\title{
Association of Tumor Necrosis Factor Receptor 1 Promoter Gene Polymorphisms (-580 A/G and -609 G/T) and TNFR1 Serum Levels with the Susceptibility to Gastric Precancerous Lesions and Gastric Cancer Related to $H$. pylori Infection in a Moroccan Population
}

\author{
Ghizlane Bounder $\mathbb{D},{ }^{1,2}$ Mohamed R. Jouimyi ${ }^{1},{ }^{1,2}$ Hasna Boura $\mathbb{D}^{1},{ }^{1}$ Hassan Jouhadi, ${ }^{3}$ \\ Wafaa Badre, ${ }^{4}$ Hakima Benomar, ${ }^{5}$ Anass Kettani ${ }^{2},{ }^{2}$ Halima Lebrazi $\mathbb{D}^{2}$ \\ and Fatima Maachi $\mathbb{D D}^{1}$ \\ ${ }^{1}$ Helicobacter Pylori and Gastric Pathologies Laboratory, Institut Pasteur du Maroc, Casablanca 20360, Morocco \\ ${ }^{2}$ Biology and Health Laboratory, Faculty of Sciences Ben M'sik, University Hassan II, Casablanca 7955, Morocco \\ ${ }^{3}$ Department of Radiotherapy Oncology, Ibn Rochd University Hospital Center, Casablanca 20250, Morocco \\ ${ }^{4}$ Gastroenterology Department, Ibn Rochd University Hospital Center, Casablanca 20250, Morocco \\ ${ }^{5}$ Histo-Cytopathology Laboratory, Institut Pasteur du Maroc, Casablanca 20360, Morocco \\ Correspondence should be addressed to Fatima Maachi; fatima.maachi@pasteur.ma
}

Received 3 July 2020; Revised 8 September 2020; Accepted 13 September 2020; Published 24 September 2020

Academic Editor: David Yang

Copyright ( 2020 Ghizlane Bounder et al. This is an open access article distributed under the Creative Commons Attribution License, which permits unrestricted use, distribution, and reproduction in any medium, provided the original work is properly cited.

\begin{abstract}
Chronic inflammation due to H. pylori infection is the risk factor of gastric cancer (GC). Through its receptor (TNFR1), TNF- $\alpha$ plays a fundamental role in inflammatory, infectious, and tumor processes. Dysregulation of TNFR1 gene expression could impact many biological processes that can lead to cancer. This study is aimed at evaluating the association of TNFR1 promoter gene polymorphisms (-580 A/G and -609 G/T) and TNFR1 serum levels with GC and precancerous lesion susceptibility. Patients suffering from gastric lesions (65 chronic gastritis, 50 precancerous lesions, and $40 \mathrm{GC}$ ) related to $H$. pylori infection and 63 healthy controls (HC) were involved in this study. Individuals are genotyped by TNFR1 gene promoter sequencing, and TNFR1 serum levels were measured by the ELISA quantitative method. Concerning TNFR1 -609 G/T locus, we noticed that the $\mathrm{T}$ allele was associated with an attenuated susceptibility to $\mathrm{GC}(\mathrm{OR}=0.4 ; p$ value $=0.02)$. At the genotypic level and under the recessive model, the TNFR1 -609 TT genotype showed a decreased risk of GC (OR $=0.3$, $p$ value $=0.03)$ compared to the combined (GG/GT) genotypes. TNFR1 serum levels have been increased together with gastric lesion severity $(p$ value $<0.05)$. The TNFR1 -609 TT genotype seemed linked to a low level of sTNFR1 compared to GT and GG genotypes $(p$ value $=0.07)$. Concerning TNFR1 -580 A/G locus, no significant relation was noticed between this polymorphism and GC susceptibility, as well as with the TNFR1 serum level. Our results suggest that the TNFR1 -609 T allele appears to have a protective effect against GC. High levels of TNFR1 serum levels seemed to be associated with the aggressiveness of gastric lesions. Therefore, our results suggest that TNFR1 -609 T/G polymorphism and the TNFR1 serum levels may be related to GC susceptibility.
\end{abstract}

\section{Introduction}

Gastric cancer is one of the most aggressive neoplasms. It is ranked among the fourth most diagnosed cancers and the second leading cause of death worldwide [1]. Gastric cancer is a multistep process, starting by Helicobacter pylori- (H. pylori-) driven inflammation, which leads to chronic gastritis, atrophic gastritis, intestinal metaplasia, and dysplasia which can then progress to an adenocarcinoma [2]. 
Chronic inflammation related to $H$. pylori infection is believed to be a critical step in gastric carcinogenesis [3]. Tumor Necrosis Factor-alpha (TNF- $\alpha$ ) is known as a principal mediator of inflammation and host response to $H$. pylori infection, and it is closely related to gastric epithelial injury $[4,5]$. Controversial functions have been attributed to this cytokine in cancer physiopathology. TNF- $\alpha$ can act as a tumor suppressor through vascular destruction, tumor necrosis, and immunostimulation. Or act as a tumor promoter by inducing cellular transformation, survival, proliferation, invasion, angiogenesis, and metastasis [6, 7]. TNF- $\alpha$ elicits its pleiotropic cellular effects through interaction with its two cell surface receptors TNFR1 and TNFR2. TNFR1 represents the primary receptor for TNF- $\alpha$ due to its ubiquitous expression and its enrolling in several biological effects [8].

The TNFR1 gene is located on chromosome $12 \mathrm{p} 13$ consisting of 10 exons, 9 introns, and a $3^{\prime}$ untranslated region. The TNFR1 gene contains a housekeeping promoter with multiple transcription start sites and CAAT box motifs and missing TATA box motifs [9]. The expression of TNFR1 is tightly regulated, in part, at the transcriptional level. Polymorphisms occurring in promoter regions of the TNFR1 gene may potentially affect the expression of TNFR1 [9]. Several studies have described some TNFR1 gene polymorphisms as sensitivity markers of inflammatory disorders and cancers [10-14].

TNFR1 is primarily produced as a transmembrane protein (tmTNFR1) consisting of 455 amino acids $(55 \mathrm{kDa})$ that gets released from the membrane by proteolytic cleavage, to generate a soluble form (sTNFR1). sTNFR1 plays an important role in the regulation of TNF- $\alpha$ activity. It can act as a TNF- $\alpha$ antagonist by competing for the interaction of TNF$\alpha$ with tmTNFR1, or as an activator through stabilizing the trimer structure of TNF- $\alpha$ by preventing its dissociation into inactive monomers, and serving as a slow-release reservoir for TNF- $\alpha$ [15]. sTNFR1 can also function as a ligand for tmTNF- $\alpha$ and induce intracellular messages via reverse signaling [16]. The serum levels of sTNFR1 and its ligand TNF- $\alpha$ are highly regulated in diverse human cancers and are suggested to be an important determinant of growth and cancer progression [7, 15, 17-19].

The identification of predictive markers for the identification and follow-up of patients that present a high risk to develop gastric cancer is the main objective of several studies. The present study is aimed at evaluating the association of TNFR1 -580 A/G and TNFR1 -609 G/T polymorphisms and sTNFR1 serum levels with gastric cancer susceptibility.

\section{Materials and Methods}

2.1. Clinical and Pathological Characteristics of the Study Population. This retrospective study focused on patients suffering from gastric disorders associated with $H$. pylori infection, collected from the Gastroenterology and Oncology Departments at the IBN ROCHD University Hospital Center from 2016 to 2018. Patients having received previous treatment for $H$. pylori eradication, proton pump inhibitors, anti-inflammatory medicines, chemotherapy or radiotherapy treatment, or suffering from any other infections, inflammatory disorders, and cancers other than distal gastric adeno- carcinoma were excluded from the study. Healthy asymptomatic subjects, with no history of gastrointestinal illnesses or regular use of any gastrointestinal and antiinflammatory medicines, were recruited from the Regional Transfusion Center of Casablanca.

All participants were informed about their inclusion in the study and agreed to it in writing form. The study protocol has been performed in accordance with the ethical standards of Helsinki and was approved by the committee of Pasteur Institute of Morocco.

Three biopsies ( 1 antrum, 1 fundus, and 1 lesser curvature) were sampled from patients admitted for endoscopy. Blood samples have been sampled from all healthy controls and patients before anesthesia and gastroscopy examination and conserved in an icebox during transport.

The biopsies were fixed in $10 \%$ formalin and taken to the Histo-Cytopathology Laboratory in Pasteur Institute of Morocco, where they were embedded in paraffin blocks, sectioned and stained in hematoxylin and eosin for conventional histopathology examination of gastric mucosal lesions and $H$. pylori infection. Classification of gastric lesions was done according to the Sydney system.

The diagnosis of $H$. pylori infection in the healthy control subjects was performed by the quantitative anti- $H$. pylori IgG ELISA method, using a commercially available kit (Euroimmun, D-23560 Lubeck (Deutschland)). A cut-off value of antibody concentration $\geq 20$ relative units $(\mathrm{RU}) / \mathrm{mL}$ was considered as positive.

2.2. Genotyping of TNFR1 -609/-580 Polymorphisms. Genomic DNA was isolated from blood taken on the EDTA tube using the commercially available kit (PureLink ${ }^{\mathrm{TM}}$ Genomic DNA Mini Kit) and stored at $-20^{\circ} \mathrm{C}$ until use.

Individuals were genotyped for TNFR1 gene promoter at position $-609 \mathrm{G} / \mathrm{T}$ and $-580 \mathrm{~A} / \mathrm{G}$ by sequencing. Firstly, the TNFR1 gene $(360 \mathrm{bp})$ was amplified from genomic DNA by PCR. The PCR was carried out in a total reaction volume of $20 \mu \mathrm{L}$, consisting of $400 \mathrm{ng}$ genomic DNA, $1 \mathrm{mM}$ of dNTPs, $3 \mathrm{mM}$ of $\mathrm{MgCl} 2,0.5 \mu \mathrm{M}$ of primers as described by Gupta et al. [11], and 1 unit of Taq polymerase (MyTaq ${ }^{\mathrm{TM}}$ Polymerase, Bioline). Thermocycling conditions of PCR were as follows: initial denaturation at $95^{\circ} \mathrm{C}$ for 1 minute, followed by 30 cycles of $95^{\circ} \mathrm{C}$ for 15 seconds, $57^{\circ} \mathrm{C}$ for 15 seconds, $72^{\circ} \mathrm{C}$ for 30 seconds, and a final extension at $72^{\circ} \mathrm{C}$ for 7 minutes. The PCR products were checked out in $1.5 \%$ agarose gel electrophoresis and purified using $1 \mu \mathrm{L}$ of exonuclease (Thermo Scientific), $1 \mu \mathrm{L}$ of alkaline phosphatase (Promega), and $5 \mu \mathrm{L}$ of PCR product and incubated at $37^{\circ} \mathrm{C}$ for 15 minutes and $80^{\circ} \mathrm{C}$ for 15 minutes. The DNA sequencing conditions were carried out based on the method as previously described [19]. The DNA sequences were read by an Applied Biosystems 377 DNA sequencer and analyzed using the BIOEDIT software package.

2.3. Measurement of sTNFR1 Serum Levels. The blood samples were taken on the serum tube, and the serum was obtained by centrifugation at $1500 \mathrm{~g}$ for $15 \mathrm{~min}$ within $1 \mathrm{~h}$ of blood collection. The serum was stored at $-80^{\circ} \mathrm{C}$ in aliquots of $500 \mu \mathrm{L}$ and melted just before testing. The measurement of sTNFR1 serum levels was performed in strict accordance 
with the kit instructions: the Human TNFR1 ELISA kit from R\&D Systems (DuoSet ELISA, USA). Total sTNFR1 concentrations in samples were expressed as $\mathrm{pg} / \mathrm{mL}$.

2.4. Statistical Analysis. The statistical program $\mathrm{R}$ software version 3.5.0 was used to carry out the statistical analysis. The descriptive data were presented in terms of frequencies. A chi-square test was used for the comparisons of genotypes and allele frequencies between cases and controls. When the number of subjects in the subgroup was lower than 10, the Fisher's correction was used. Odds Ratios (OR), as well as their $95 \%$ confidence intervals (CI), were computed to estimate the risk of gastric lesions with TNFR1 variants. The normality of distribution was assessed by the Shapiro-Wilk test, and then, a comparison of quantitative data between two groups was performed by the Wilcoxon test. The comparison of quantitative data between more than two groups was performed by the Kruskal-Wallis test. The differences were regarded as significant at $p$ value $<0.05$.

\section{Results}

3.1. Clinical and Pathological Characteristics of the Study Population. A total of 218 participants with $H$. pylori-positive status, composed of 63 asymptomatic healthy control subjects (HC) and 155 patients with various gastric lesions, 65 with chronic gastritis, 50 with precancerous lesions (30 with atrophic gastritis, 20 with intestinal metaplasia), and 40 with gastric cancer, were enrolled in the present study. Twentyfour patients and $4 \mathrm{HC}$ were excluded.

The male/female ratio in the case group and the control group was, respectively, $73 / 82$ and 34/29. The mean age of HC was $43 \pm 8$, chronic gastritis was $45 \pm 16$, precancerous lesions was $53 \pm 14$, and gastric cancer was $56 \pm 14$ years.

3.2. Genotyping of TNFR1 -609/-580 Polymorphisms. Regarding TNFR1 $-609 \mathrm{G} / \mathrm{T}$ locus, the frequency of T allele is $37.3 \%$ in $\mathrm{HC}$ and has shown a decrease with the severity of gastric lesions, with the following order: $36.2 \%$ in chronic gastritis, $31 \%$ in precancerous lesions, and $21.3 \%$ in gastric cancer. The $\mathrm{T}$ allele was observed to be associated with an attenuated risk of gastric cancer with an $\mathrm{OR}=0.4(p$ value $=0.02)$ compared to the G allele (Table 1). Genotypically, we noticed that the TT genotype was lower in patients with gastric cancer (10\%) and precancerous lesions (16\%) compared to patients suffering from chronic gastritis (23.1\%) and HC (28.6\%). But statistically, the alteration remains not significant $(p$ value $=$ 0.07 ). According to the different dominance models of the TNFR1 -609 T allele, we noticed that in cases of dominance and codominance, the combined genotypes (TT+GT) reduced the risk of gastric cancer, but statistically, the results were not significant $(\mathrm{OR}=0.5,95 \%$ CI $0.2-1.2$, $p$ value $=0.2$ ). While under the recessive model, we noticed a significantly reduced risk for gastric cancer $(\mathrm{OR}=0.3,95 \% \mathrm{CI}$ 0.09-0.9, $p$ value $=0.03)$ in subjects with the TNFR1 -609 TT genotype compared to the combined genotypes (GG+GT). The distribution of alleles and genotypes for the TNFR1 -609 G/T locus in the patient groups and controls is shown in Table 1.
Regarding the TNFR1 $-580 \mathrm{~A} / \mathrm{G}$ locus, there were no significant differences in allele and genotype distributions between patients and HC. The distribution of alleles and genotypes for the TNFR1 -580 locus in the patient groups and controls is shown in Table 1.

3.3. Measurement of sTNFR1 Serum Levels. The serum levels of sTNFR1 were observed to be increased together with the severity of gastric lesions. The lowest levels were recorded in controls with an average value of $779.8 \mathrm{pg} / \mathrm{mL}$ and have shown an increase with the severity of gastric lesions, with the following order: $881.3 \mathrm{pg} / \mathrm{mL}$ in patients with chronic gastritis, $892.6 \mathrm{pg} / \mathrm{mL}$ in those with precancerous lesions, and $929.9 \mathrm{pg} / \mathrm{mL}$ recorded among patients suffering from gastric cancer (Table 2).

A disparity in the sTNFR1 serum levels between all the groups of patients and HC was noted and was statistically significant ( $p$ value $<0.05$ ). However, no significant difference was observed between chronic gastritis, PL, and gastric cancer (Table 2).

3.4. Association between TNFR1 -609/-580 Polymorphisms and sTNFR1 Serum Levels. Regarding the TNFR1 -609 G/T locus, the mean level of sTNFR1 in GG $(872.1 \mathrm{pg} / \mathrm{mL})$ and GT $(875.6 \mathrm{pg} / \mathrm{mL})$ genotypes was approximately the same, while a relatively low value of sTNFR1 was observed in the TT genotype $(820.4 \mathrm{pg} / \mathrm{mL}$ ) (Table 3$)$. It appears that individuals with the TT genotype tend to express low sTNFR1 levels compared to those with GT and GG genotypes, but the difference remains statistically not significant ( $p$ value $=0.07$ ).

Regarding the TNFR1 -580 A/G locus, no difference in the distribution of sTNFR1 serum levels according to the genotypes was revealed (Table 3). The mean level of sTNFR1 was, respectively, $866.2,852.5$, and $826.71 \mathrm{pg} / \mathrm{mL}$ in individuals with AA, GA, and GG genotypes.

\section{Discussion}

The genetic predisposition to cancers has been demonstrated through several studies and is still a subject of interest and scientific research $[11,13,19]$. Serum levels of sTNFR1 are used as a predictive marker of multifactorial inflammatory disorders and cancers $[12,17,20,21]$. The present study was designed to evaluate the potential use of TNFR1 genotyping (TNFR1 -580 A/G and TNFR1 -609 G/T) and TNFR1 serum levels as predictive markers in the identification and follow-up of patients that present a high risk to develop gastric cancer.

The association of TNFR1 $-609 \mathrm{G}>\mathrm{T}$ and TNFR1 -580 A $>$ G polymorphisms with the susceptibility to gastric cancer and precancerous lesions in our population was evaluated.

Concerning the TNFR1 -609 G/T locus, it seemed that the frequency of the T allele and TT genotype decreased with the severity of the gastric lesions. The $\mathrm{T}$ allele was observed to be associated with an attenuated risk of gastric cancer with an $\mathrm{OR}=0.4$ ( $p$ value $=0.02)$ compared to the $\mathrm{G}$ allele. The TNFR1 -609 TT genotype was associated with a reduced risk of gastric cancer $(\mathrm{OR}=0.3,95 \% \mathrm{CI} 0.09-0.9, p$ value $=0.03)$ 
TABLE 1: TNFR1 promoter polymorphisms at positions -609 and -580 in patients with gastric lesions and healthy controls.

\begin{tabular}{|c|c|c|c|c|}
\hline & $\begin{array}{c}\mathrm{HC} \\
N(\%)\end{array}$ & $\begin{array}{c}\text { CG } \\
N(\%)\end{array}$ & $\begin{array}{c}\mathrm{PL} \\
N(\%)\end{array}$ & $\begin{array}{c}\mathrm{GC} \\
N(\%)\end{array}$ \\
\hline \multicolumn{5}{|l|}{ TNFR1 -609 G/T genotypes } \\
\hline GG & $34(54.0)$ & $33(50.8)$ & $27(54)$ & $27(67.5)$ \\
\hline GT & $11(17.4)$ & $17(26.1)$ & $15(30)$ & $9(22.5)$ \\
\hline TT & $18(28.6)$ & $15(23.1)$ & $8(16)$ & $4(10.0)$ \\
\hline$p$ value* & & 0.47 & 0.15 & 0.07 \\
\hline OR, $[95 \% \mathrm{CI}], p$ value (TT+GT vs. GG) & & $1,[0.5-2.1], 0.86$ & $0.9,[0.42-1.9], 0.8$ & $0.5,[0.2-1.3], 0.2$ \\
\hline OR, $[95 \% \mathrm{CI}], p$ value $(\mathrm{GG}+\mathrm{GT}$ vs. TT+GT) & & $0.9,[0.5-1.9], 1$ & $0.8,[0.4-1.7], 0.7$ & $0.5,[0.2-1.2], 0.2$ \\
\hline OR, $[95 \% \mathrm{CI}], p$ value (GG+GT vs. TT) & & $0.7,[0.3-1.6], 0.5$ & $0.4,[0.1-1.2], 0.2$ & $0.3,[0.09-0.9], 0.03$ \\
\hline \multicolumn{5}{|l|}{ TNFR1 $-609 \mathrm{G} / \mathrm{T}$ alleles } \\
\hline G & $79(62.7)$ & $83(63.8)$ & $69(69)$ & $63(78.7)$ \\
\hline $\mathrm{T}$ & $47(37.3)$ & $47(36.2)$ & $31(31)$ & $17(21.3)$ \\
\hline$p$ value* & & 0.85 & 0.32 & 0.01 \\
\hline OR, $[95 \% \mathrm{CI}], p$ value (T vs. $\mathrm{G}$ ) & & $0.9,[0.6-1.6], 0.89$ & $0.7,[0.4-1.3], 0.33$ & $0.4,[0.2-0.8], 0.02$ \\
\hline \multicolumn{5}{|l|}{ TNFR1 -580 A/G genotypes } \\
\hline AA & $45(71.4)$ & $55(84.6)$ & $33(66)$ & $33(82.5)$ \\
\hline AG & $16(25.4)$ & $9(13.8)$ & $17(34)$ & $6(15)$ \\
\hline GG & $2(3.2)$ & $1(1.6)$ & $0(0)$ & $1(2.5)$ \\
\hline$p$ value* & & 0.17 & 0.42 & 0.42 \\
\hline OR, $[95 \% \mathrm{CI}], p$ value $(\mathrm{GA}+\mathrm{GG}$ vs. $\mathrm{AA})$ & & $0.4,[0.2-1.1], 0.08$ & $1.3,[0.6-2.9], 0.54$ & $0.5,[0.2-1.4], 0.24$ \\
\hline OR, $[95 \% \mathrm{CI}], p$ value $(\mathrm{AA}+\mathrm{GA}$ vs. $\mathrm{GG}+\mathrm{GA})$ & & $0.5,[0.2-1.2], 0.1$ & $1.1,[0.5-2.5], 0.8$ & $0.6,[0.2-1.6], 0.36$ \\
\hline OR, $[95 \% \mathrm{CI}], p$ value $(\mathrm{AA}+\mathrm{GA}$ vs. $\mathrm{GG})$ & & $0.5,[0.04-5.4], 0.6$ & $0,[0-0], 0.5$ & $0.6[0.07-8.9], 1$ \\
\hline \multicolumn{5}{|l|}{ TNFR1 -580 A/G alleles } \\
\hline A & $106(84.1)$ & $119(93)$ & $83(83)$ & $72(90)$ \\
\hline G & $20(15.9)$ & $11(7)$ & $17(17)$ & $8(10)$ \\
\hline$p$ value* & & 0.08 & 0.82 & 0.23 \\
\hline OR, $[95 \% \mathrm{CI}] ; p$ value (G vs. A) & & $0.5,[0.2-1], 0.08$ & $1.1,[0.5-2.2], 0.85$ & $0.6,[0.2-1.4], 0.29$ \\
\hline
\end{tabular}

HC: healthy controls; CG: chronic gastritis; PL: precancerous lesions; GC: gastric cancer. ${ }^{*} p$ values were calculated using the chi-squared test or Fisher test.

TABLE 2: Association between sTNFR1 serum levels and gastric lesion severity.

\begin{tabular}{|c|c|c|c|c|}
\hline sTNFR1 $(\mathrm{pg} / \mathrm{mL})$ & $\mathrm{HC}$ & CG & $\mathrm{PL}$ & GC \\
\hline Mean & 779.8 & 881.3 & 892.6 & 929.9 \\
\hline Median & 780 & 881 & 921 & 881 \\
\hline SD & 105.1 & 158 & 154.6 & 196.7 \\
\hline Range & $540-958$ & $622-1257$ & $520-1132$ & 633-1439 \\
\hline$p$ value significance intragroups ${ }^{*}$ & \multicolumn{4}{|c|}{0.000018} \\
\hline \multicolumn{5}{|l|}{$p$ value significance intergroups ${ }^{* *}$} \\
\hline Case vs. controls & & 0.0003 & 0.00003 & 0.0002 \\
\hline Case vs. chronic gastritis & & - & 0.36 & 0.28 \\
\hline Case vs. precancerous lesions & & - & - & 0.61 \\
\hline
\end{tabular}

HC: healthy controls; CG: chronic gastritis; PL: precancerous lesions; GC: gastric cancer; *: Kruskal-Wallis test; **: Wilcoxon test.

compared to the combined genotypes $(\mathrm{GG}+\mathrm{GT})$, under the recessive model.

Our finding is consolidated with several studies that reported the association of this polymorphism with a reduced risk of oral, colon, and gastric cancer and other inflammatory pathologies such as invasive pulmonary asper- gillosis [10-13]. However, other reports have shown the association of TNFR -609 $\mathrm{T}$ allele with a high risk of hepatic cancer, Crohn's, and ankylosing spondylitis diseases [2224]. The effect of this polymorphism on cancer pathologies is still unknown, so further studies are needed to clarify this association. 
TABLE 3: Association between TNFR1 -609 G/T and -580 A/G locus genotypes and sTNFR1 serum levels.

\begin{tabular}{|c|c|c|c|c|c|c|}
\hline \multirow{2}{*}{ TNFR1 (pg/mL) } & \multicolumn{3}{|c|}{ TNFR1 -609 G/T genotypes } & \multicolumn{3}{|c|}{ TNFR1 -580 A/G genotypes } \\
\hline & GG & GT & TT & AA & AG & GG \\
\hline Range & $520-1272$ & $630-1251$ & $540-1129$ & $520-1272$ & $680-1237$ & $540-1112$ \\
\hline Mean & 872.1 & 875.6 & 820.4 & 866.2 & 852.5 & 826.7 \\
\hline SD & 163.9 & 153.1 & 141.7 & 162.1 & 138.3 & 249.2 \\
\hline$p$ value significance intragroups* & & 0.15 & & & 0.85 & \\
\hline \multicolumn{7}{|l|}{$p$ value significance intergroups ${ }^{* *}$} \\
\hline Case vs. TNFR1 -609 GG & & 0.97 & 0.07 & & & \\
\hline Case vs. TNFR1 -609 GT & & & 0.07 & & & \\
\hline Case vs. TNFR1 -580 AA & & & & & 0.65 & 0.83 \\
\hline Case vs. TNFR1 -580 AG & & & & & & 0.7 \\
\hline
\end{tabular}

*: Kruskal-Wallis test; **: Wilcoxon test.

Concerning the TNFR1 -580 A/G locus, statistically, no alterations in the genotypic and allelic distributions between groups were noted ( $p$ value $>0.05$ ). This result is consolidated by other studies reporting the absence of the association between TNFR1 $-580 \mathrm{~A} / \mathrm{G}$ polymorphism and gastric pathologies, chronic pancreatitis, rheumatoid arthritis, and Kawasaki disease $[10,25,26]$.

Determination of sTNFR1 levels in several body fluids, including serum, provides valuable information on a variety of pathological conditions [27]. In this study, a significant disparity of sTNFR1 serum levels between cases and controls was noticed. Compared to HC, we remarked that sTNFR1 serum levels increase among patients with chronic gastritis, precancerous lesions, and mainly among those suffering from gastric cancer. Our results suggest a positive association between the increase of sTNFR1 serum levels and gastric carcinogenesis.

H. pylori infection provokes the production of inflammatory mediators, including TNF- $\alpha$, IL8, IL1, MMPs, and growth factors $[28,29]$. These mediators can activate the expression of the TNFR1 gene and its release $[16,21]$. Shibata et al. showed an elevation of sTNF- $\alpha$ and sTNFR1 in $H$. pylori-infected patients compared to uninfected patients [30]. Regarding cancer diseases, increased levels of circulating sTNFR1 have been reported in patients suffering from several cancers $[7,15,27]$. As regards gastric cancer, Shibata et al. revealed that high serum levels of sTNFR1 were associated with the severity of gastric cancer (studied population: 42 gastric cancer patients compared to $15 \mathrm{HC}$ ) [31]. Aderka et al.'s study showed that TNFR1 serum levels were higher in patients with gastric cancer than those in controls (studied population: 3 patients compared to $42 \mathrm{HC}$ ) [32]. Several studies suggest that high levels of sTNFR1 in patients are associated with poor prognosis, more aggressive immunological response, high severity of cancer, advanced tumor stages, a reduced response to treatment, high mortality, and short survival $[7,17,20,21,33,34]$, while other studies reported that a high level of sTNFR1 reflects the antitumor defense and could be used to estimate the immune potential of cancer patients $[35,36]$.

The polymorphisms located in the promoter region of the TNFR1 gene could have a significant impact on tran- scriptional regulation. Our findings revealed that individuals carrying TNFR1 -609 TT genotype tend to express low sTNFR1 levels compared to those carrying TNFR1 -609 GT and GG genotypes, but these observations remain statistically insignificant ( $p$ value $=0.07$ ). Previous studies have reported the repression impact of this polymorphism on TNFR1 expression $[9,23]$. TNFR1 $-609 \mathrm{~T}$ allele was associated with an attenuated risk of the gastric cancer group and was linked to low TNFR1 levels. We can suggest that the elevation of circulating sTNFR1 is implicated in the promotion of gastric cancer and not in its regression. Regarding the TNFR1 -580 A/G locus, no alteration in sTNFR1 serum levels according to the genotypes of this locus was recorded. This finding is supported by Wang et al. who demonstrated the absence of the impact of TNFR1 -580 A/G polymorphism on TNFR1 production [37]. Further research on a larger population is needed to clarify the effect of these polymorphisms.

\section{Conclusions}

In summary, the TNFR1 $-609 \mathrm{~T}$ allele seems to be linked to an attenuated susceptibility to gastric cancer. A gradual increase in serum levels of sTNFR1 with the severity of gastric carcinogenesis lesions was demonstrated. TNFR1 -609 TT genotype appears to be associated with reduced sTNFR1 serum levels. Regarding the TNFR1-580 A/G locus, no significant association with a gastric cancer risk or its impact on sTNFR1 serum levels was recorded. Further studies including a large population and diverse ethnicities are needed to illumine the association of TNFR $1-609 \mathrm{G} / \mathrm{T}$ and $-580 \mathrm{~A} / \mathrm{G}$ polymorphisms and sTNFR1 serum levels with gastric cancer susceptibility, to be used as potential markers for the identification and follow-up of patients at a high risk of developing gastric cancer.

\section{Data Availability}

The data used to support the findings of this study are included within the article. 


\section{Disclosure}

This work is part of an approved thesis.

\section{Conflicts of Interest}

The authors declare that there is no conflict of interest regarding the publication of this paper.

\section{Acknowledgments}

We acknowledge the contributions of our patients' partners, and we ask God for the recovery and good health of the patients. We also acknowledge the team of the HistoCytopathology Laboratory, Institut Pasteur du Maroc, Casablanca, Morocco; Dr. Meriem Khyati from the Laboratory of Onco-Virology, Institut Pasteur du Maroc; Pr Ibrahimi from the Faculty of Medicine and Pharmacy of Rabat; Pr Rachid Saile from the Faculty of Sciences Ben M'sik; and Dr. Abdeljabbar Majd from the Regional Transfusion Center of Casablanca. This work was supported by the Pasteur Institute of Morocco.

\section{References}

[1] R. Sitarz, M. Skierucha, J. Mielko, J. Offerhaus, R. Maciejewski, and W. Polkowski, "Gastric cancer: epidemiology, prevention, classification, and treatment," Cancer Management and Research, vol. 10, pp. 239-248, 2018.

[2] A. Koulis, A. Buckle, and A. Boussioutas, "Premalignant lesions and gastric cancer: current understanding," World Journal of Gastrointestinal Oncology, vol. 11, no. 9, pp. 665678, 2019.

[3] K. A. Bockerstett and R. J. DiPaolo, "Regulation of gastric carcinogenesis by inflammatory cytokines," Cellular and Molecular Gastroenterology and Hepatology, vol. 4, no. 1, pp. 47-53, 2017.

[4] R. Rivalino, D. Darmadi, and G. A. Siregar, "Correlation between TNF- $\alpha$ and degree of gastritis," The Indonesian Journal of Gastroenterology, Hepatology, and Digestive Endoscopy, vol. 19, no. 1, pp. 16-19, 2018.

[5] T. Yamamoto, M. Kita, T. Ohno, Y. Iwakura, K. Sekikawa, and J. Imanishi, "Role of tumor necrosis factor-alpha and interferon-gamma in Helicobacter pylori infection," Microbiology and Immunology, vol. 48, no. 9, pp. 647-654, 2004.

[6] S. F. Josephs, T. E. Ichim, S. M. Prince et al., "Unleashing endogenous TNF-alpha as a cancer immunotherapeutic," Journal of Translational Medicine, vol. 16, no. 1, p. 242, 2018.

[7] I. Martínez-Reza, L. Díaz, and R. García-Becerra, "Preclinical and clinical aspects of TNF- $\alpha$ and its receptors TNFR1 and TNFR2 in breast cancer," Journal of Biomedical Science, vol. 24, no. 1, p. 90, 2017.

[8] L. Cabal-Hierro and P. S. Lazo, "Signal transduction by tumor necrosis factor receptors," Cellular Signalling, vol. 24, no. 6, pp. 1297-1305, 2012.

[9] S. V. Sennikov, F. F. Vasilyev, J. A. Lopatnikova, N. S. Shkaruba, and A. N. Silkov, "Polymorphisms in the tumor necrosis factor receptor genes affect the expression levels of membrane-bound type I and type II receptors," Mediators of Inflammation, vol. 2014, Article ID 745909, 11 pages, 2014.
[10] G. Bounder, A. Essadik, H. Jouhadi et al., "TNF- $\alpha$ receptor 1 promoter gene polymorphisms and susceptibility to gastric cancer related to $H$. pylori infection in Moroccan population," International Journal of Science and Research (IJSR), vol. 6, no. 6, p. 5, 2017.

[11] R. Gupta, S. C. Sharma, and S. N. Das, "Association of TNF- $\alpha$ and TNFR1 promoters and $3^{\prime}$ UTR region of TNFR2 gene polymorphisms with genetic susceptibility to tobacco-related oral carcinoma in Asian Indians," Oral Oncology, vol. 44, no. 5, pp. 455-463, 2008.

[12] J. Sainz, I. Salas-Alvarado, E. López-Fernández et al., “TNFR1 mRNA expression level and TNFR1 gene polymorphisms are predictive markers for susceptibility to develop invasive pulmonary aspergillosis," International Journal of Immunopathology and Pharmacology, vol. 23, no. 2, pp. 423-436, 2010.

[13] M. L. Slattery, A. Lundgreen, K. L. Bondurant, and R. K. Wolff, "Tumor necrosis factor-related genes and colon and rectal cancer," International Journal of Molecular Epidemiology and Genetics, vol. 2, no. 4, pp. 328-338, 2011.

[14] J. Sode, U. Vogel, S. Bank et al., "Anti-TNF treatment response in rheumatoid arthritis patients is associated with genetic variation in the NLRP3-inflammasome," PloS One, vol. 9, no. 6, article e100361, 2014.

[15] L. Puimège, C. Libert, and F. Van Hauwermeiren, "Regulation and dysregulation of tumor necrosis factor receptor-1," Cytokine \& Growth Factor Reviews, vol. 25, no. 3, pp. 285-300, 2014.

[16] Y. Dong, D. Dekens, P. de Deyn, P. Naudé, and U. Eisel, “Targeting of tumor necrosis factor alpha receptors as a therapeutic strategy for neurodegenerative disorders," Antibodies, vol. 4, no. 4, pp. 369-408, 2015.

[17] M. S. Ahluwalia, S. Bou-Anak, M. E. Burgett et al., "Correlation of higher levels of soluble TNF-R1 with a shorter survival, independent of age, in recurrent glioblastoma," Journal of Neuro-Oncology, vol. 131, no. 3, pp. 449-458, 2017.

[18] B. Kotowicz, J. Kaminska, M. Fuksiewicz et al., "Clinical significance of serum CA-125 and soluble tumor necrosis factor receptor type I in cervical adenocarcinoma patients," International Journal of Gynecologic Cancer, vol. 20, no. 4, pp. 588-592, 2010.

[19] G. Bounder, M. R. Jouimyi, H. Boura et al., "Associations of the $-238(\mathrm{G} / \mathrm{A})$ and $-308(\mathrm{G} / \mathrm{A})$ TNF- $\alpha$ promoter polymorphisms and TNF- $\alpha$ serum levels with the susceptibility to gastric precancerous lesions and gastric cancer related to Helicobacter pylori infection in a Moroccan population," Asian Pacific Journal of Cancer Prevention, vol. 21, no. 6, pp. 1623-1629, 2020.

[20] F. Vinante, A. Rigo, C. Tecchio et al., "Serum levels of p55 and p75 soluble TNF receptors in adult acute leukaemia at diagnosis: correlation with clinical and biological features and outcome," British Journal of Haematology, vol. 102, no. 4, pp. 1025-1034, 2002.

[21] A. C. Carlsson, C. C. Juhlin, T. E. Larsson et al., "Soluble tumor necrosis factor receptor 1 (sTNFR1) is associated with increased total mortality due to cancer and cardiovascular causes - findings from two community based cohorts of elderly," Atherosclerosis, vol. 237, no. 1, pp. 236-242, 2014.

[22] S. Bek, J. V. Nielsen, A. B. Bojesen et al., "Systematic review: genetic biomarkers associated with anti-TNF treatment response in inflammatory bowel diseases," Alimentary Pharmacology \& Therapeutics, vol. 44, no. 6, pp. 554-567, 2016.

[23] S. Kim, S.-M. Moon, Y. S. Kim et al., "TNFR1 promoter -329G/T polymorphism results in allele-specific repression of TNFR1 expression," Biochemical and Biophysical Research Communications, vol. 368, no. 2, pp. 395-401, 2008. 
[24] S. Zhao, H. Chen, G. Wu, and C. Zhao, "The association of NLRP3 and TNFRSF1A polymorphisms with risk of ankylosing spondylitis and treatment efficacy of etanercept," Journal of Clinical Laboratory Analysis, vol. 31, no. 6, article e22138, 2017.

[25] S. L. Bridges, G. Jenq, M. Moran, T. Kuffner, W. C. Whitworth, and J. McNicholl, "Single-nucleotide polymorphisms in tumor necrosis factor receptor genes: definition of novel haplotypes and racial/ethnic differences," Arthritis and Rheumatism, vol. 46, no. 8, pp. 2045-2050, 2002.

[26] A. Schneider, K. Pogue-Geile, M. M. Barmada, E. Myers-Fong, B. S. Thompson, and D. C. Whitcomb, "Hereditary, familial, and idiopathic chronic pancreatitis are not associated with polymorphisms in the tumor necrosis factor $\alpha$ (TNF- $\alpha$ ) promoter region or the TNF receptor 1 (TNFR1) gene," Genetics in Medicine, vol. 5, no. 2, pp. 120-125, 2003.

[27] K. Hosono, E. Yamada, H. Endo et al., "Increased tumor necrosis factor receptor 1 expression in human colorectal adenomas," World Journal of Gastroenterology, vol. 18, no. 38, pp. 5360-5368, 2012.

[28] N. Bagheri, L. Salimzadeh, and H. Shirzad, "The role of T helper 1-cell response in Helicobacter pylori-infection," Microbial Pathogenesis, vol. 123, pp. 1-8, 2018.

[29] B. M. Mahdi, "Immune response to Helicobacter pylori," Trends Helicobacter Pylori Infection, vol. 79, 2014.

[30] J. Shibata, H. Goto, T. Arisawa et al., "Regulation of tumour necrosis factor (TNF) induced apoptosis by soluble TNF receptors in Helicobacter pylori infection," Gut, vol. 45, no. 1, pp. 24-31, 1999.

[31] M. Shibata, M. Takekawa, and S. Amano, "Increased serum concentrations of soluble tumor necrosis factor receptor I in noncachectic and cachectic patients with advanced gastric and colorectal cancer," Surgery Today, vol. 28, no. 9, pp. 884-888, 1998.

[32] D. Aderka, H. Englemann, V. Hornik et al., "Increased serum levels of soluble receptors for tumor necrosis factor in cancer patients," Cancer Research, vol. 51, no. 20, pp. 5602-5607, 1991.

[33] R. Jain, L. Poisson, J. Narang et al., "Correlation of perfusion parameters with genes related to angiogenesis regulation in glioblastoma: a feasibility study," American Journal of Neuroradiology, vol. 33, no. 7, pp. 1343-1348, 2012.

[34] M. Onsrud, A. Shabana, R. Austgulen, and K. Nustad, "Comparison between soluble tumor necrosis factor receptors and CA125 in peritoneal fluids as a marker for epithelial ovarian cancer," Gynecologic Oncology, vol. 57, no. 2, pp. 183-187, 1995.

[35] R. A. Burger, K. M. Darcy, P. J. DiSaia et al., “Association between serum levels of soluble tumor necrosis factor receptors/CA 125 and disease progression in patients with epithelial ovarian malignancy," Cancer, vol. 101, no. 1, pp. 106-115, 2004.

[36] E. Jablonska, M. Kiluk, L. Piotrowski, Z. Grabowska, W. Markiewicz, and J. Jablonski, "Tumor necrosis factoralpha and soluble tumor necrosis factor receptors in the culture supernatants of polymorphonuclear cells and peripheral blood mononuclear cells from cancer patients," European Cytokine Network, vol. 9, no. 2, pp. 155-159, 1998.

[37] G.-B. Wang, C.-R. Li, J. Yang, P.-Q. Wen, and S.-L. Jia, “A regulatory polymorphism in promoter region of TNFR1 gene is associated with Kawasaki disease in Chinese individuals," Human Immunology, vol. 72, no. 5, pp. 451-457, 2011. 